\title{
Antimicrobial and antioxidant surface modification toward a new silk-fibroin (SF)-L-Cysteine material for skin disease management
}

\author{
Frederico Nogueira ${ }^{\mathrm{a}, \mathrm{b}}$, Luíza Granadeiro ${ }^{\mathrm{a}}$, Claudia Mouro ${ }^{\mathrm{b}}$, Isabel C. Gouveia ${ }^{\mathrm{b}, *}$ \\ a CICS-UBI - Health Sciences Research Centre, University of Beira Interior, Portugal \\ ${ }^{\mathrm{b}}$ FibEnTech - Fiber Materials and Environmental Technologies, University of Beira Interior, Portugal
}

\section{A R T I C L E I N F O}

\section{Article history:}

Received 7 September 2015

Received in revised form 1 December 2015

Accepted 21 December 2015

Available online 23 December 2015

\section{Keywords:}

L-Cysteine (L-Cys)

Antimicrobial applications

Medical textiles

Atopic dermatitis (AD)

Staphylococcus aureus

\begin{abstract}
A B S T R A C T
A novel dressing material - silk fibroin fabric (SF)-L-Cysteine ( $\mathrm{L}-\mathrm{Cys}$ ) - is here developed to be used as standard treatment for atopic dermatitis (AD), which combines comfort, thermic, and tensile strength properties of silk materials with antioxidant and antimicrobial effects of L-Cys. A careful understanding about the linking strategies is needed in order not to compromise the bioavailability of L-Cys and deplenish its bioactivity. Durability was also addressed through washing cycles and compared with hospital requirements, according to international Standard EN ISO 105-C06:2010. The present research also analyze the interactions between Staphylococcus aureus and SF-L-Cys under simulating conditions of AD and demonstrated the effectiveness of a double covalent grafting, with the importance of SF tyrosine (Tyr) covalent linkage with L-Cys (SF-g-L-Cys/Tyr-g-L-Cys) even after several washing cycles, twenty five, whereas for a disposable application a single covalent mechanism of grafting L-Cys proved to be sufficient (SF-g-L-Cys). Results showed effective antimicrobial activities exhibiting higher inhibition ratios of 98.65\% for SF-g-L-Cys after 5 washing cycles, whereas 97.55\% for SF-g-L-Cys/Tyr-g-L-Cys after 25 washing cycles, both at pH 9.5 grafting strategy. Furthermore, it is also reported a non-protumoral effect of L-Cys. A new advance is herein achieved at the world of medical antimicrobial textiles tailored to address wound moisture environment and exudate self-cleaning, which may open novel applications as complementary therapy for $\mathrm{AD}$ disease.
\end{abstract}

(c) 2015 Elsevier B.V. All rights reserved.

\section{Introduction}

Silk, produced by silkworm Bombyx morii, consists of fibroin and sericin. Fibroin, which is the structural protein of silk fibers, comes out from the silkworm gland in the form of filaments enclosed in a water-soluble sericin gluelike coating [1]. Silk fibroin (SF) is composed of heavy $(350 \mathrm{kDa})$ and light chain $(25 \mathrm{kDa})$ polypeptides linked by a disulfide bond [2,3]. Its structure is mainly composed by the aminoacids glycine (46\%), alanine (29\%), and serine $(12 \%)$, which are aminoacids having small side chains. Also, SF presents tyrosine (4.8\%), which has an aromatic side chain [4]. They form flexible and stretching resistant secondary structure beta-sheets, which render silk its characteristic mechanical strength [5-7]. Silk is non-toxic, non-immunogenic, biocompatible, and hydrophilic. Although it is stated that the itching of $A D$ is exacerbated by spiky

\footnotetext{
* Corresponding author at: FibEnTech R\&D - Fiber Materials and Environmental Technologies, University of Beira Interior, Rua Marquês d'Ávila e Bolama, 6201-001 Covilhã, Portugal.

E-mail address: igouveia@ubi.pt (I.C. Gouveia).
}

fibers of wool and by the wild texture of cotton [8,9], silk, on the other hand, with its extremely smooth and its comfortable properties reduces these effects and therefore has been increasingly researched for applications related to atopic dermatitis (AD). Furthermore, Sugihara et al. [10] postulated that wounds treated with a silk dressing healed 7 days faster, by promoting the enhanced synthesis of collagen and epithelialization [10].

$\mathrm{AD}$, also known as eczema, is a chronic and recurrent inflammatory pruritic skin disorder which causes lichenification, papules, and excoriations. AD has been increasing over the past decades, reaching prevalence of about $20 \%$ in children and $3 \%$ in adults worldwide [11]. A combination of genetic, environmental, and immunological factors may be the main causes for the development of $\mathrm{AD}$, whose consequences can be psychologically traumatic, leading to emotional stress and decreasing substantially the quality of life. AD is characterized by a combination of T-helper type (Th) 2 immune activation, skin infection/colonization by Staphylococcus aureus, and skin barrier dysfunction. AD prevalence rise may be explained by today's lifestyle and nutrition [11]. The cleaner environment in upbringing as well as antibiotics abusing during early childhood has induced more often Th 2 immune responses, 
which are related to allergic responses, instead of Th 1 immune responses, which respond to infections [12]. Likewise, immune system responds to superantigens of cell walls of $S$. aureus inducing IgE allergic response [13]. After an AD trauma, an uncontrolled rising in body temperature and dehydration can lead to heart failure and death [14]. AD is a 2-step pathogenesis. First an allergic antigen-antibody (IgE) reaction takes place, and then a microbial infection takes over and maintains or exacerbates the disease [15]. $\mathrm{AD}$ patients often have more $S$. aureus ratio on the skin, diseased and normal, when comparing to non-diseased individuals. Furthermore, wounded skin can be colonized up to $1000 \times$ more S. aureus when compared to normal skin [16].

S. aureus is surrounded by a capsule that inhibits phagocytosis by polimorphonuclear leukocytes, and a slime layer composed of monosaccharides, peptides, and proteins, which allow its attachment to different tissue receptors and other external devices, such as catheters, suture and prosthesis. Also, S. aureus produces a variety of toxins that work as virulent factors [17]. Clinical diseases are triggered by $S$. aureus producing toxins or direct invasion and destruction of tissues. Notwithstanding that clinical manifestations of disease rely almost exclusively on toxin activity, in minor situations it can also be due to bacterial proliferation that forms abscess and tissue destruction. It also presents resistance against the currently used antimicrobials [18].

In the present work we have chosen L-Cysteine (L-Cys), which is a $\alpha$-amino acid with a thiol group at the end of its variable side group, which is nucleophilic and has a wide spectrum of bioactivity [19], relying its activity on metabolic disturbance and membrane depolarization [20]. It targets the bacterial membrane and causes a great decrease in enzymatic activity and bacterial metabolism. Owing to its ability to form conjugates with free radicals or trace elements [21], L-Cys may also render our final application antioxidant properties.

This research work consisted on the development of two processes that allowed the covalent grafting of L-Cys onto SF: (i) functionalization of SF carboxylates (degraded amide groups) and cross-linking with the amine compounds of L-Cys; (ii) functionalization of SF Tyr with L-Cys, with the formation of Schiff base intermediates that were chemically stabilized by reductive amination.

This allowed to draw important conclusions regarding the best way to pursuit for a disposable application, as well as when designed for a multi-patient with laundry-resistant capabilities (after 25 washing cycles).

\section{Materials and methods}

\subsection{Materials}

Silk was obtained from the cocoons of larvae of the silkworm $B$. morii (Brazil). Silk was knitted at a rectilinear needle loom with 7 Picks per inch, with a Jersey knit structure and a linear density of 21.68 number metric (Nm). Its sericin was removed with dichloromethane in a soxhlet apparatus. Silk fibroin (SF) was then functionalized in a L-Cysteine (L-Cys) (Sigma-Aldrich) solution $\left(\mathrm{ddH}_{2} \mathrm{O}\right)$ at pH's 5.5 and 9.5. Covalent bonds at tyrosine (Tyr) were performed with $\mathrm{NaCNBH}_{3}$ dissolved in $\mathrm{NaOH}: \mathrm{HCl}$ buffers (Panreac). S. aureus (ATCC 6538) strain was grown on Nutrient Agar (NA) and Nutrient Broth (NB) (Panreac).

\subsection{Methods}

\subsubsection{Minimal inhibitory concentration (MIC) evaluation}

Minimal inhibitory concentration (MIC) determination was performed with the microdilution method in accordance with M07-A6 guidelines applied to S. aureus (ATCC 6538) - Standard JIS L 1902:2002. Briefly, L-Cys was serial diluted in Mueller-Hinton Broth (MHB) (Sigma-Aldrich) to yield concentrations from 0.3 to $20 \mathrm{mg} / \mathrm{mL}$. Overnight liquid $S$. aureus cultures were turbidityadjusted to $0.5 \mathrm{McF}$ arland $\left(1 \times 10^{7} \mathrm{CFU} / \mathrm{mL}\right.$ for S. aureus $)$ with sterile $\mathrm{H}_{2} \mathrm{O}$, from which $500 \mu \mathrm{L}$ were blended with $4500 \mu \mathrm{L}$ MHB, forming the bacterial work suspension. $50 \mu \mathrm{L}$ of work suspensions L-Cys dilutions and stock solution - were added into 96-multi well plate. Samples were incubated for $24 \mathrm{~h}$ at $37^{\circ} \mathrm{C}$. Deposited microorganisms (dot-shaped) in the bottom of each well were examined. The last well in the dilution series that showed deposit (microbial killing) corresponded to MIC of the antimicrobial agent. Triplicates were used.

\subsubsection{Antioxidant activity: determination of DPPH}

(2,2-diphenyl-1-picrylydrazyl) radical scavenging activity

The antioxidant activity of L-Cys was determined by the method of radical scavenging activity using $\mathrm{DPPH}^{\circ}$. $\mathrm{DPPH}^{\circ}$ is stable nitrogen radical that can accept an electron or hydrogen radical to become a stable diamagnetic molecule. $\mathrm{DPPH}^{\circ}$ bears a deep purple color, but over the course of the reaction becomes colorless, when the odd electron of its nitrogen atom is reduced by receiving a hydrogen atom from the antioxidant testing compound L-Cys. The $\mathrm{DPPH}^{\circ}$ assay was adapted according to the procedure described by [22]. Briefly, the stock solution was prepared by dissolving $4 \mathrm{mg}$ DPPH with $100 \mathrm{~mL}$ methanol. The percentage of remaining $\mathrm{DPPH}^{\circ}$ for each concentration of L-Cys was determined using the equation of the obtained calibration curve and absorbance values measured at $517 \mathrm{~nm}$, at $30 \mathrm{~min}$ time point:

$\% \mathrm{DPPH}^{\circ}{ }_{\mathrm{REM}}=\left[\mathrm{DPPH}^{\circ}\right]_{\mathrm{T}} /\left[\mathrm{DPPH}^{\circ}\right]_{\mathrm{T}=0} \times 100 \%$

Efficient concentration $\left(\mathrm{EC}_{50} \mu \mathrm{g} / \mathrm{mL}\right.$ ) was defined as substrate concentration to produce $50 \%$ reduction of the $\mathrm{DPPH}^{\circ}$.

\subsubsection{Tumoral activity assay}

The cytotoxicity of L-Cys was performed, adapting the procedures from Standard E DIN EN ISO 10993-5. It was selected the concentration of L-Cys chosen for this work, $1 \%$, and its half $0.5 \%$, on breast cancer cell line (NCI-PBCF-HTB22 (ATCC ${ }^{\circledR} \mathrm{HTB}-22^{\mathrm{TM}}$ )). Incubation was done at $37^{\circ} \mathrm{C}$ for $48 \mathrm{~h}$ under a humidified atmosphere containing $5 \% \mathrm{CO}_{2}$. MTT viability assay was performed. Solvent, positive and negative controls were included.

\subsubsection{Silk substrate preparation}

2.2.4.1. Silk material. $20 \mathrm{~g}$ of SF knitted fabric was boiled for $4 \mathrm{~h}$ in dichloromethane in a soxhlet apparatus to extract residual lipids, and then rinsed thoroughly with distilled water. It was then washed according to BS 4923: 1991 - methods for individual domestic washing and drying for use in textile testing, in a $4 \mathrm{~g} / \mathrm{L}$ solution of ECE Reference Detergent (the formulation of the detergent is given in BS 1006:CO6 (ISO 105:CO6)) for $60 \mathrm{~min}$ at $40^{\circ} \mathrm{C}$, rinsed, and dried afterwards at $30^{\circ} \mathrm{C}$ until used, in order to remove impurities and soils.

2.2.4.2. Grafting/functionalization processes. SF knitted fabric samples were functionalized by means of an exhaustion process at $90{ }^{\circ} \mathrm{C}, 15 \mathrm{rpm}$, in a Mathis-BFA12 finishing machine. Single covalent strategy (SF-g-L-Cys): samples were immersed in a $1 \%$ over the weight of the fiber (owf) L-Cys solution $\left(\mathrm{ddH}_{2} \mathrm{O}\right)$ adjusted to pH's 5.5 and 9.5, for $3 \mathrm{~h}$. Fig. 1 A. Double covalent strategy (SF-g-LCys/Tyr-g-L-Cys): samples were immersed in a $1 \%$ over the weight of the fiber (owf) L-Cys solution $\left(\mathrm{ddH}_{2} \mathrm{O}\right)$ adjusted to $\mathrm{pH}$ 's 5.5 and 9.5, for $3 \mathrm{~h}$, and were added $0.3(\mathrm{w} / \mathrm{V}) \mathrm{NaCNBH}_{3}$ solution from the beginning of second hour. Samples were rinsed three times with dd $\mathrm{H}_{2} \mathrm{O}$ and dried at $30^{\circ} \mathrm{C}$ (Fig. 1B). 


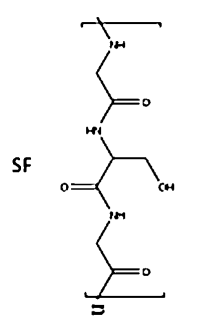

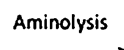
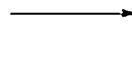

SF.g.L.CyS

SF

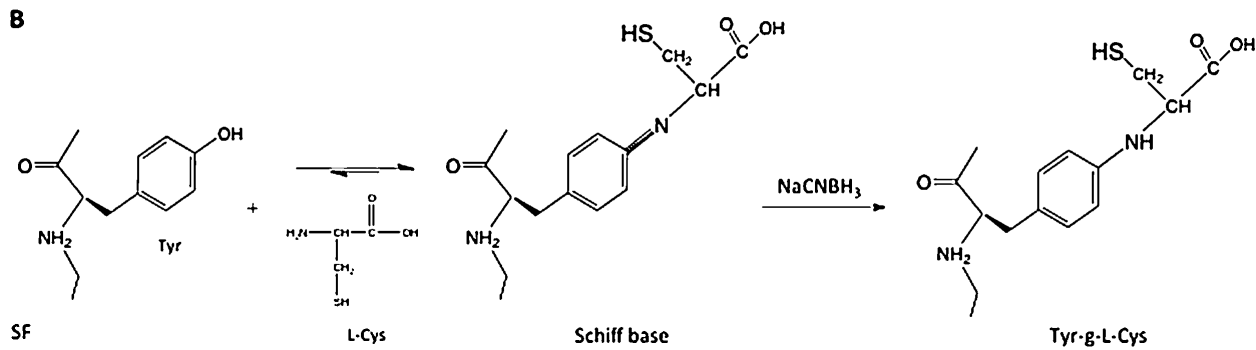

Fig. 1. Strategies for the activation of silk fibroin. (A) SF-g-L-Cys; (B) SF-g-L-Cys/Tyr-g-L-Cys.

Firstly, SF knitted fabric samples were functionalized on its carboxylates (degraded amide groups) and were cross-linked with the amine compounds present in L-Cys solution (SF-g-L-Cys). Secondly, SF knitted fabric samples were functionalized on its Tyr ketone groups, with the formation of Schiff base intermediates that were chemically stabilized by reductive amination, forming ultimately a covalent bond (SF-g-L-Cys/Tyr-g-L-Cys).

2.2.4.3. Washing cycles assay - resistance to laundry. Samples were (i) washed in a $4 \mathrm{~g} / \mathrm{L}$ solution of AATCC 1993 Standard Reference Detergent WOB for 5 washing cycles performed at $40^{\circ} \mathrm{C}$ during $30 \mathrm{~min}$ each, and (ii) washed in a $4 \mathrm{~g} / \mathrm{L}$ solution of AATCC 1993 Standard Reference Detergent WOB for 25 washing cycles performed at $40^{\circ} \mathrm{C}$ during $30 \mathrm{~min}$ each. These methods were adapted from the international standard EN ISO 105-C06:2010. Washing cycles were done prior to all studies in order to assess the resistance to washing of L-Cys bonds on SF surface.

\subsubsection{Grafted-silk characterization}

SF samples were characterized using different surface analyzing techniques against appropriate controls.

2.2.5.1. Contact angle. Contact angles were measured using an OCAH-200 set-up by Dataphysics equipped with a high-speed video system with CCD video camera with a resolution of $752 \times 484$.

2.2.5.2. Fourier transform infrared spectroscopy (FT-IR). The chemical composition of SF, SF-g-L-Cys, and SF-g-L-Cys/Tyr-g-L-Cys was analyzed by means of FT-IR. After three rinses (no washing cycles), 5 washing cycles, and 25 washing cycles FT-IR spectra were analyzed. Measurements were done with a Thermo-Nicolet is10 FTIR spectrophotometer. Each sample was scanned 64 times, with a spatial frequency resolution of $4 \mathrm{~cm}^{-1}$.

2.2.5.3. Exhaustion rates: degree of functionalization. In order to assess the efficiency of the functionalization, i.e. the quantity of L-Cys that the substrate SF was able to crosslink, exhaustion rate of functionalization assays were performed for each condition. Ellman's reagent, (5,5'-dithio-bis-(2-nitrobenzoic acid/DTNB) (Sigma) produces a yellow color when bonded to free thiol groups in solution. After reacting with free thiol groups (of L-Cys) there is formation of a dissulphydric compound and a colored species 2-nitro-5-thiobenzoic acid (TNB). This colorimetric assay was performed by measuring and comparing the functionalization solution before and after functionalization, after doing a calibration with known concentrations of L-Cys. For each sample $250 \mu \mathrm{L}$ was added to a test tube containing $2.5 \mathrm{~mL}$ of sodium phosphate $(\mathrm{pH} 8)$ and $50 \mu \mathrm{L}$ of Ellman's reagent. Test tubes were vortexed and were incubated for 15 minutes before their absorbance being read at $412 \mathrm{~nm}$.

2.2.5.4. Tensile strength assays. In order to evaluate the degradation caused on SF by the functionalization processes, several quality control assays were performed. Resistance to rupture assays were done in all samples, with and without L-Cys, to subsequent comparison. Assays were performed on a Dynamometer, according to Standard EN ISO 2062, at $20 \pm 2{ }^{\circ} \mathrm{C}$, under $60 \%$ relative humidity. Samples were strip-cut $5 \mathrm{~cm} \times 50 \mathrm{~cm}$, also string cut, and placed between dynamometer tweezers. A defined pre-tension was set in the beginning, and the test ended up with samples rupture.

\subsubsection{Antimicrobial activity assessment}

The antimicrobial behavior of SF samples functionalized with L-Cys (-g-L-Cys and -g-L-Cys/Tyr-g-L-Cys) was tested according to Japanese Industrial Standard JIS L 1902:2002, specific for textile samples. Gram-positive S. aureus (ATCC 6538) strain was chosen once it plays an important role in atopic dermatitis (AD). Briefly, inoculum of $S$. aureus was prepared with a final concentration of $1-5 \times 10^{6}$, bacteria/mL. $200 \mu \mathrm{L}$ of $S$. aureus were inoculated on $0.4 \mathrm{~g}$ square samples. Samples were incubated for $24 \mathrm{~h}$. Antimicrobial activity was assessed by a quantitative method to determine the percentage of bacterial growth inhibition (\% of Inhibition) at 24 hours:

\%Inhibition $=\frac{C-A}{C} \times 100$

where $C$ represents the average value of Colony Forming Units $(\mathrm{CFU}-\mu \mathrm{g} / \mathrm{mL}$ ) of non-modified $\mathrm{SF}$, and A represents the average value of CFU of grafted SF. 
Table 1

Concentration of L-Cys versus \% of remaining [DPPH].

\begin{tabular}{lc}
\hline Concentration $\mu \mathrm{g} / \mathrm{mL}$ & \% of remaining $[\mathrm{DPPH}]$ \\
\hline 0 & 100 \\
0.05 & 8.459185758 \\
0.04 & 47.30720708 \\
0.03 & 48.37492768 \\
0.02 & 76.5334419 \\
0.1 & 4.770788268 \\
EC50 & 0.029957282 \\
\hline
\end{tabular}

\subsubsection{Scanning electron microscopy (SEM)}

Adsorbed bacteria were reticulated with $1.5 \%$ glutaraldehyde overnight at $4{ }^{\circ} \mathrm{C}$. Samples were then dehydrated with an increasing ethanol/water gradient (50-99\%, v/v), and subjected to critical point drying (K850, EMITECH). After being sputtered with a thin layer of gold, samples were examined by SEM (Hitachi S2700).

Images were formed through secondary electrons using a high voltage of $20 \mathrm{kV}$. Five fields per sample were obtained using a magnification of 20000 and $75000 \times$.

\subsubsection{Transmission electron microscopy (TEM)}

The morphology of adhered S. aureus on SF knitted fabric functionalized with L-Cys (-g-L-Cys and -g-L-Cys/Tyr-g-L-Cys) was addressed by TEM. Bacteria were stained with $2 \%$ uranyl acetate for 5 min, and mounted on a mesh with thin bar copper grids covered with formvar. Each sample was examined at $30000 \times$ magnification using HITACHI HT7700 TEM at an accelerating voltage of $80 \mathrm{kV}$.

\subsubsection{Statistical analysis}

SPSS Statistics 21.0 software was used to perform the statistical analysis of the data using One-Way ANOVA, with Tukey as Post Hoc tests for establishing multiple comparisons between samples in this study. Values of $p<0.05$ were considered statistically significant.

\section{Results}

\subsection{Minimal inhibitory concentration (MIC) of L-Cys}

Minimal inhibitory concentration (MIC) of L-Cysteine (L-Cys) against $S$. aureus was found to be $5 \mathrm{mg} / \mathrm{mL}$.

\subsection{Antioxidant activity}

\subsubsection{Determination of DPPH (2,2-diphenyl-1-picrylydrazyl) radical scavenging activity}

The reduction capability of the $\mathrm{DPPH}^{\circ}$ radical was determined by the decrease on its absorbance at $517 \mathrm{~nm}$, induced by Cysteine. Table 1 shows that as the concentration of Cysteine increased, the percentage of remaining $\mathrm{DPPH}^{\circ}$ diminished.

The percentage of remaining $\mathrm{DPPH}^{\circ}$ was proportional to the antioxidant concentration of L-Cys. The concentration that caused a decrease in the initial $\mathrm{DPPH}^{\circ}$ concentration by $50 \%$ (EC50) was found to be $0.029957 \mu \mathrm{g} / \mathrm{mL}$, which implies that L-Cysteine have antioxidant activity.

\subsection{Tumoral activity assay}

Soluble L-Cys did not show any effect on breast cancer cell line MCF-7. Values were not significantly different from control, with $4 \%$ and $9 \%$ of growth above at doses of 1 and $0.5 \mathrm{mg} / \mathrm{mL} \mathrm{L-Cys,} \mathrm{respec-}$ tively (Fig. 2). Only an alteration above 30\% in comparison with controls is considered pro-tumorigenic [23].

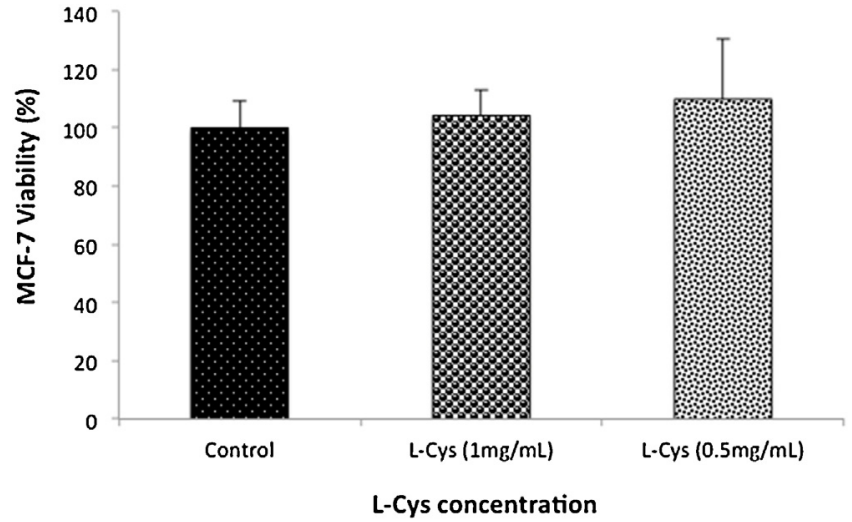

Fig. 2. MCF-7 breast cancer cell line viability percentage when in contact with two concentrations of L-Cys and the untreated controls.

\subsection{Silk characterization}

\subsubsection{Contact angle}

The wettability of silk fibroin (SF) was assessed by the measurement of the contact angle between the surface and water using the sessile drop technique. Water contact angles measured at $10 \mathrm{~s}$ showed a hydrophilic SF surface; $21.1 \pm 1.5^{\circ}$. Therefore grafted SF samples proved to be able to absorb exudates and provide wound moisture, which render them usable as healing wound dressings against $\mathrm{AD}$.

\subsubsection{Fourier transform infrared spectroscopy (FT-IR)}

The infrared spectra of unmodified SF is shown in Fig. 3a. Peaks at $3300 \mathrm{~cm}^{-1}$ and $3100 \mathrm{~cm}^{-1}$ are associated with amides $A$ and $B$, respectively. At $1650 \mathrm{~cm}^{-1}$ vibrations are due to $C=0$ stretching vibrations of amide I, and the peak at $1520 \mathrm{~cm}^{-1}$ is related to $\mathrm{N}-\mathrm{H}$ in plane bending together with the out-of-phase $\mathrm{C}-\mathrm{N}$ stretching of amide II.

SF covalent structural modification after 25 washing cycles was also assessed by FT-IR. IR spectra confirmed L-Cys bonded on SF surface, as shown by the increase of peaks associated with L-Cys immobilization on a surface, around 3300,3100 and $1650 \mathrm{~cm}^{-1}$, as shown in Fig. 3B. Amide I is related to vibrations of the peptide bonds. However, the peak at $1520 \mathrm{~cm}^{-1}$ related to amide II diminishes, which tells the information of SF protein secondary structure modified/unfolding based on the extent of L-Cys crosslinking to SF. Furthermore $3300 \mathrm{~cm}^{-1}$ is also associated with $\mathrm{OH}$ stretching related to free $-\mathrm{OH}$ in $\mathrm{SF}$ aromatic tyrosine [24]. When covalent bond formed at Tyr residue the vibration assigned to $-\mathrm{OH}$ decreased. This might be the reason why the sample SF-gL-Cys/Tyr-g-L-Cys at pH $9.590^{\circ} \mathrm{C}$ (spectrum e) showed significant decrease of the absorption band at $3300 \mathrm{~cm}^{-1}$.

\subsubsection{Efficiency of functionalization/grafting}

The exhaustion efficiency of L-Cys on SF fabric was assessed after $3 \mathrm{~h}$ of functionalization at $90^{\circ} \mathrm{C}$. All samples gave rise to figures of over $80 \%$ exhaustion rate, once L-Cys readily adsorbed to SF chemistry. SF-g-L-Cys/Tyr-g-L-Cys double covalent bonding showed $80.35 \%$ (pH 5.5) and 80.71\% (pH 9.5), whereas SF-g-L-Cys single covalent bonding rates were $87.09 \%(\mathrm{pH} 5.5)$ and $85.94 \%(\mathrm{pH}$ 9.5).

\subsubsection{Tensile strength assays}

Tensile tests were done to evaluate the effect of single covalent and double covalent treatments on the mechanical properties of samples, and are shown in Table 2.

A worthy maximum stress and elongation were observed for SF thread and strip at different conditions of functionalization; Results 
A

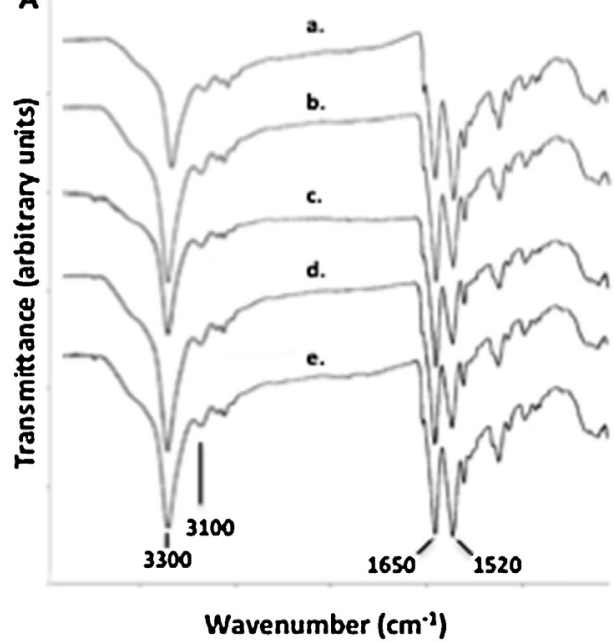

B

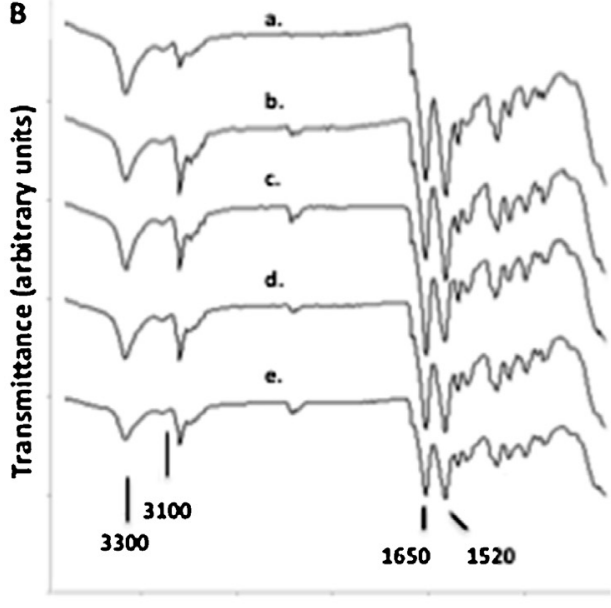

Wavenumber $\left(\mathrm{cm}^{-1}\right)$

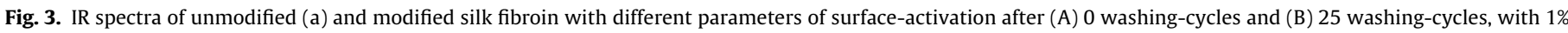

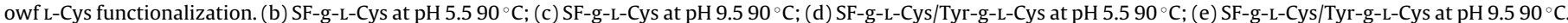

Table 2

Mechanical characterization. Resistance to rupture assays in thread and strip.

\begin{tabular}{llll}
\hline \multirow{5}{*}{ Thread } & Material & $\begin{array}{l}\text { Stress at break } \\
\text { (Newton) }\end{array}$ & Elongation (\%) \\
& SF & $31.08 \pm 4.06$ & $15.37 \pm 4.15$ \\
& pH 5.5 SF-g-L-Cys & $28.59 \pm 4.28$ & $13.69 \pm 3.45$ \\
& pH 5.5 SF-g-L-Cys/Tyr-g-L-Cys & $27.10 \pm 5.83$ & $13.99 \pm 4.16$ \\
& pH 9.5 SF-g-L-Cys & $33.69 \pm 0.36$ & $18.14 \pm 0.02$ \\
& pH 9.5 SF-g-L-Cys/Tyr-g-L-Cys & $31.15 \pm 12.7$ & $16.49 \pm 6.72$ \\
Strip & SF & $620.7 \pm 54.9$ & $57.49 \pm 4.75$ \\
& pH 5.5 SF-g-L-Cys & $640.6 \pm 55.2$ & $59.15 \pm 4.47$ \\
& pH 5.5 SF-g-L-Cys/Tyr-g-L-Cys & $486.6 \pm 80.5$ & $55.25 \pm 4.37$ \\
& pH 9.5 SF-g-L-Cys & $566.0 \pm 46.3$ & $58.02 \pm 8.39$ \\
& pH 9.5 SF-g-L-Cys/Tyr-g-L-Cys & $709.3 \pm 52.2$ & $64.13 \pm 6.24$ \\
\hline
\end{tabular}

were not significantly different from controls, which proves the covalent modifications set up in this work did not compromise SF.

\subsection{Antimicrobial assays}

\subsubsection{Quantitative method (suspension)}

SF activated surfaces showed good microbial inhibition results, as shown in Fig. 4. Following three rinses after functionalization, no significant differences $(p<0.05)$ between covalent/double covalent treatments could be seen regarding microbial killing, except that all of them were highly microbicidal. After 24 hours of incubation, results of microbial reduction were close to $100 \%$.

After 5 washing cycles, SF-g-L-Cys treatment at pH 9.5 showed to be significantly $(p<0.05)$ more microbicidal for $S$. aureus $-98.65 \%$ - against $89.33 \%$ of SF-g-L-Cys pH 5.5. Also, SF-g-L-Cys/Tyr-g-LCys modifications were $77.38 \%$ for functionalization at $\mathrm{pH} 9.5$, and $78.40 \%$ for $\mathrm{pH} 5.5$.

On the other hand, after 25 washing cycles SF-g-L-Cys/Tyr-g-LCys double covalent modification proved to be worthwhile, once it showed such a great amount of microbial reduction, which proved to form bonds/structural conformations strong enough to resist to several washings. S. aureus when adsorbed to SF-g-L-Cys/Tyr-g-LCys, modified under pH 9.5 with $1 \%$ owf L-Cys, showed a significant CFU reduction of $97.55 \%$ ( $p<0.05$ ), whereas when adsorbed to SFg-L-Cys bacterial reduction was not so prominent, with results averaging $67.39 \%$.

\subsection{Scanning electron microscopy (SEM)}

For any condition after 25 washing cycles, there was a decrease in the number of S. aureus adsorbed. However, for SF-g-L-Cys/Tyr-gL-Cys functionalized at $\mathrm{pH}$ 9.5, an outstanding killing effect showed almost no bacteria adsorbed to fabric surface (Fig. 5).

\subsection{Transmission electron microscopy (TEM)}

Following 25 washing cycles, Fig. 6 shows that there was a fierce degradation of $S$. aureus membranes, when adhered to SF-g-LCys/Tyr-g-L-Cys, especially at pH 9.5 (Fig. 6e). Considering the effect of SF-g-L-Cys/Tyr-g-L-Cys modified at pH 5.5 (Fig. 6c), it showed adhered bacteria with their membranes slightly harmed, when comparing to controls.

\section{Discussion}

This work compared single covalent against double covalent immobilization of a bioactive agent L-Cysteine ( $\mathrm{L}$-Cys) on silk fibroin (SF) knitted fabric to originate an effective antimicrobial wound dressing (bioactive material) against atopic dermatitis (AD) disease. Furthermore, end applications of laundry resistance versus disposibility, are herein discussed.

L-Cys amino acid, which has a wide spectrum of microbial activity and well-known safety [10-12], was for the first time functionalized at its lowest concentration - $1 \%$ over the weight of the fabric (wof). In previous works the minimum amount used with efficiency of microbial killing was $5 \%$ owf, in combination with either wool [23], or cotton [10,12]. This L-Cys fall is understandable, in that recently new approaches relying on removal of lipids from the surface of fibers have exposed their surface with a variety of reactive moieties. In this sense, covalent treatments can be directed straightforward to these reactive sites, rather than physically-directed, which offers higher durability and resistance to laundry [25].

For L-Cys immobilization onto SF fabric two processes were considered in order to potentiate its activity. The first process relied on modification of $87 \%$ of SF structure, namely the covalent bonding on glycine, alanine, and serine amino acids. L-Cys was blended with SF at both low and high pH's. The strong acid $\mathrm{HCl}$ plus heat $\left(90^{\circ} \mathrm{C}\right)$, as well as strong base $\mathrm{NaOH}$ plus heat $\left(90^{\circ} \mathrm{C}\right)$, made SF amides hydrolize to carboxylic acids through reverse of Fischer 


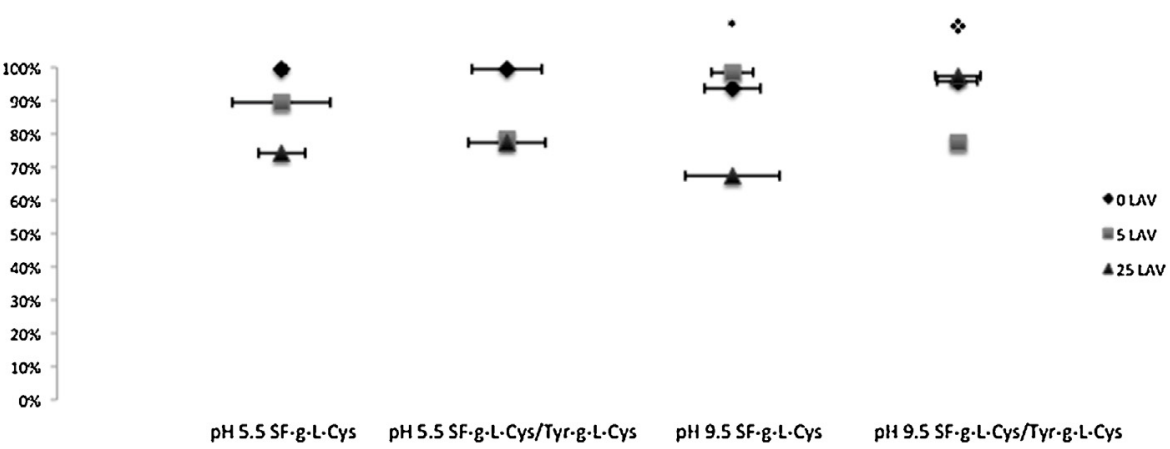

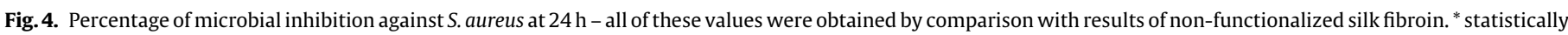
significant for 5 washing cycles. statistically significant for 25 washing cycles $(p<0.05)$.
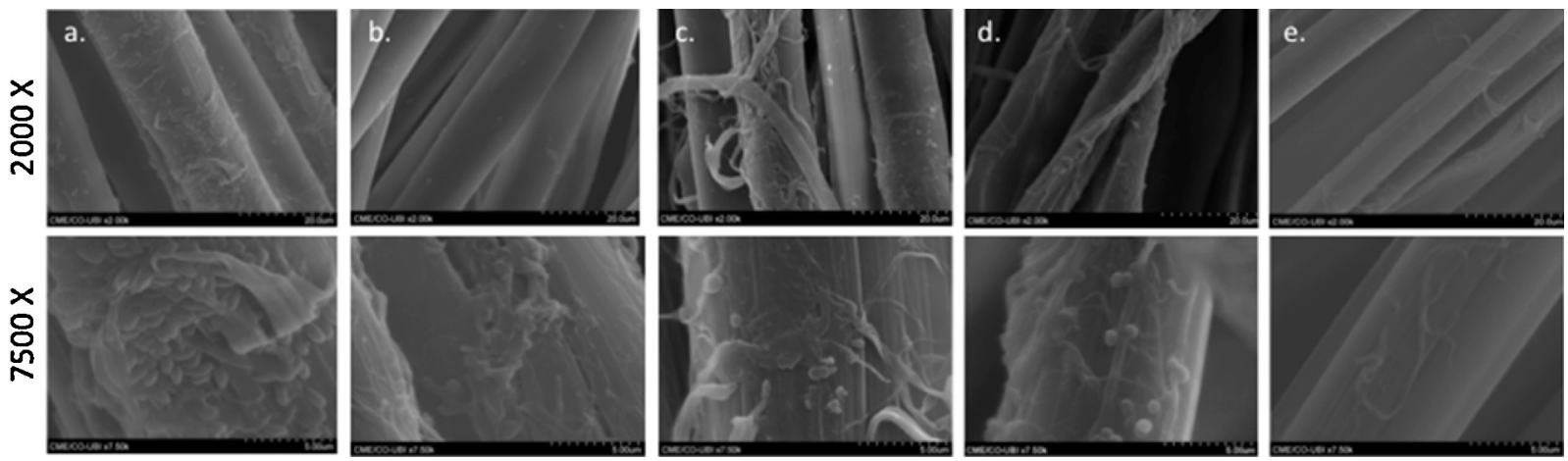

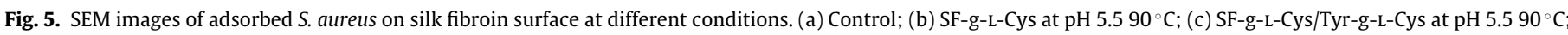
(d) SF-g-L-Cys at pH $9.590^{\circ} \mathrm{C}$; (e) SF-g-L-Cys/Tyr-g-L-Cys at pH $9.590^{\circ} \mathrm{C}$.
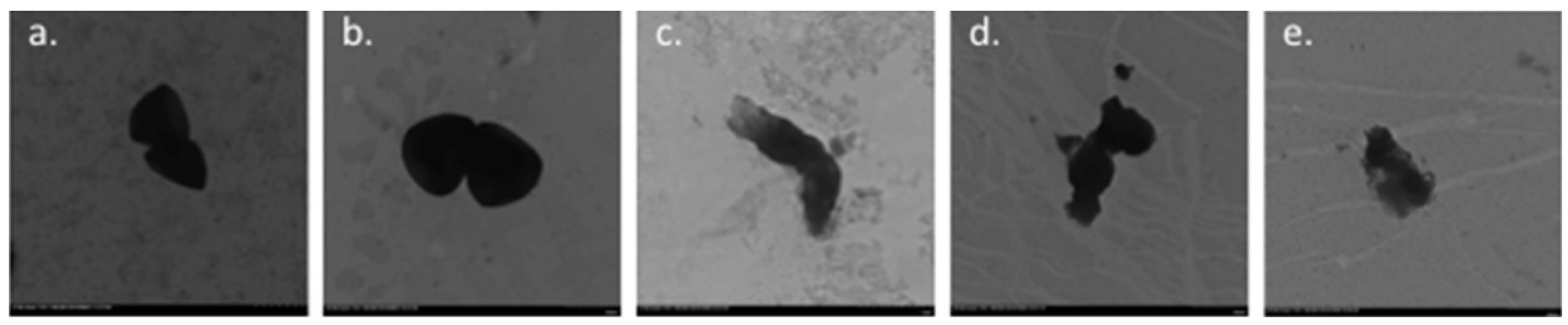

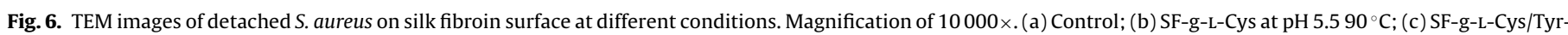
g-L-Cys at pH $5.590^{\circ} \mathrm{C}$; (d) SF-g-L-Cys at pH $9.590^{\circ} \mathrm{C}$; (e) SF-g-L-Cys/Tyr-g-L-Cys at pH $9.590^{\circ} \mathrm{C}$.

esterification and "saponification", respectively. Also, the presence of further carboxylates in SF is also described in literature [26]. Amino groups of L-Cys were then covalently linked by reacting with these carboxylic acids by aminolysis (SF-g-L-Cys). The second process relied on covalent modification of further $4.8 \%$ of SF structural tyrosine aromatic side chain. Aldehydes of tyrosine side chains were blended with the amines of L-Cys, forming unstable Schiff base intermediates (or imines). These intermediates were chemically stabilized by reductive amination with $\mathrm{NaCNBH}_{3}$ [27], forming ultimately a covalent bond. In the first process, Schiff base intermediates also formed at these sites but reverted, once they were not stabilized by reduction. Furthermore, in this process of grafting L-Cys on Tyr, important issues as Tyr localization, and its influence on backbone conformation and packing were regarded [4]. L-Cys carries a thiolate group in its molecular structure which kills microorganisms through disulfide bridge establishment with some bacterial wall structural components [28] or even intracellular components if internalized.

The ability of L-Cys to inhibit the growth of microorganisms in solution was given by Minimal Inhibitory Concentration (MIC) studies of soluble L-Cys against $S$. aureus which was $5 \mathrm{mg} / \mathrm{mL}$. This value is high when compared with chitosan polymer $(0.05 \mathrm{mg} / \mathrm{mL})$ and, on the other side, lower than triclosan $(6 \mathrm{mg} / \mathrm{mL})[29,30]$. When we compare these former MIC results with regard of settling the same molecule (L-Cys) on a surface, data demonstrated immobilization advantageous with the requirement of lower amount of L-Cys, namely $1 \%$ owf ( $1 \%$ over the weight of the fiber (owf) in a ratio of $1 \mathrm{~g}$ fiber $/ 50 \mathrm{~mL}$ solution; thus, $0.2 \mathrm{mg} / \mathrm{mL}$ ). L-Cys molecules in solution bind and gather each other through S-S bonds, losing their main reactive thiolates. On the other side, immobilizing L-Cys onto a surface prevented its agglomeration and allowed the use of lesser quantity to coat the surface, in order to grant SF fibers antibacterial activity.

It has already been demonstrated that topical application of antioxidants decreased wound healing time [31]. The total antioxidant capacity of L-Cys was assessed by using spectrophotometric methods with $\mathrm{DPPH}^{\circ}$ (2,2-diphenyl-1-picrylydrazyl) [22]. The LCys concentration that caused a decrease in the initial $\mathrm{DPPH}^{\circ}$ by $50 \%$ $\left(\mathrm{EC}_{50}\right)$ was found to be $0,029957 \mu \mathrm{g} / \mathrm{mL}$. These results showed that L-Cys has antioxidant activity. Sánchez-Moreno [32] showed EC50 values of a few other antioxidants, as gallic acid $(26 \mu \mathrm{g} / \mathrm{mL})$, tannic $(59 \mu \mathrm{g} / \mathrm{mL})$, caffeic acid $(69 \mu \mathrm{g} / \mathrm{mL})$, ascorbic acid $(76 \mu \mathrm{g} / \mathrm{mL})$, 
queratin $(84 \mu \mathrm{g} / \mathrm{mL})$, BHA $(93 \mu \mathrm{g} / \mathrm{mL})$ and rutin $(102 \mu \mathrm{g} / \mathrm{mL})$. These values show that albeit L-Cys is antioxidant, its antioxidant power is low but can be increased by rising the L-Cys concentration, if necessary (customized).

The concentration of L-Cys that was used for SF functionalization did not interfere with tumoral cells growth rate, which proves the safety of using this application in neoplastic patients. Furthermore, L-Cys has already been studied for its cytotoxicity against fibroblasts, proving to be safe, hence not cytotoxic [20].

SF hydrophilic surface, with a contact angle of $21.1 \pm 1.5^{\circ}$, besides absorbing AD wound exudate and providing wound hydration which opens the way to important factors for proper healing, showed to attract $S$. aureus present in an AD disease environment, allowing $S$. aureus to contact and be eliminated by the grafted LCys. J. Ji and W. Zhang stated that a hydrophilic surface attracted $S$. aureus [33] whereas H. Yang and Y. Deng indicated that a hydrophobic surface reduced its adhesion [34].

The formation of silk structure is driven by Tyr residues, which have a strong effect not only locally but also long-range intermolecular interactions [4]. Upon its double functionalization with L-Cys, SF structure might have adopted such an altered folding due to covalent bonding at Tyr residue. This might be the reason why the sample SF-g-L-Cys/Tyr-g-L-Cys functionalized at pH 9.5 $90^{\circ} \mathrm{C}$ showed significant decrease of the FT-IR absorption band at $3300 \mathrm{~cm}^{-1}$. Nevertheless, it is possible to conclude that the diffusing capacity of L-Cys in the fiber was high, $>80 \%$, which means L-Cys has the ability to bind covalently to the fiber surface easily and strongly. Also, high exhaustion rates meant a low quantity of L-Cys required to achieve an effective antimicrobial activity.

Accordingly, FT-IR results confirmed that L-Cys was well immobilized to SF fibers at both pH's of functionalization. Furthermore, as washing cycles were performed, effects at 5 and 25 washing cycles showed SF-g-L-Cys/Tyr-g-L-Cys grafting to be multiple washing resistant along with SF-g-L-Cys grafting at $\mathrm{pH}$ 9.5. In accordance with the chemical surface analyses of SF fiber surfaces, microbiologic data after $24 \mathrm{~h}$ of incubation, showed for 5 washing cycles the highest killing of bacteria (98.65\%) happened at pH 9.5 through L-Cys grafted on a single covalent way (SF-gL-Cys) $(p<0.05)$. This high microbicidal reduction might be due to the structural/conformational accessibility of L-Cys on SF when comparing to SF-g-L-Cys/Tyr-g-L-Cys. Furthermore, after 25 washing cycles the optimal bacterial killing effect (97.55\%) happened at $\mathrm{pH} 9.5$ through double covalent grafting SF-g-L-Cys/Tyr-g-L-Cys $(p<0.05)$. This high microbicidal reduction might be related to the exposing of erstwhile structural folding-protected L-Cys due to multiple washing cycles. A qualitative analysis was also performed through Scanning Electron Microscopy (SEM) and Transmission Electron Microscopy (TEM) by characterizing $S$. aureus according to its shape when adsorbed on SF surface after it had been washed 25 times (25 washing cycles). Data demonstrated that few bacteria adhered to the background fibers and the ones adhered were in a bad shape/morphology at the condition of SFg-L-Cys/Tyr-g-L-Cys functionalized at $\mathrm{pH}$ 9.5. As expected, acidic conditions of functionalization yielded poorer grafting of L-Cys on SF fibers. On the other hand, according to Hornsey and Pepper [35] the higher $\mathrm{pH}$ condition of functionalization led to higher effectiveness of grafting [35] once protonated amines of L-Cys were more attracted to the SF negative carboxylate groups when under the alkaline $\mathrm{pH}$ 9.5. Also, SF high exhaustion of LCys is due to its structural reactivity, which translated in higher retention after several washing cycles, leading to increased antimicrobial properties of SF fibers, when compared to cotton [20]. Mechanical properties of SF showed high, and were not significantly altered with any treatments in this study, which means SF structure never got compromised by used functionalization processes.

\section{Conclusions}

This research work describes both single covalent and double covalent grafting methods for the functionalization of silk fibroin (SF) with antibacterial and antioxidant agent L-Cysteine (L-Cys). The developed SF-L-Cys materials have the potential to inhibit medically relevant $S$. aureus bacterial species and as such to be used to control atopic dermatitis since no toxicity was found. Moreover, the durability of the fabric was significantly improved when the active antibacterial agent L-Cys was double covalently attached to SF tyrosine. It was demonstrated the effectiveness of a double covalent grafting, with the importance of SF tyrosine (Tyr) covalent linking with L-Cys (SF-g-L-Cys/Tyr-g-L-Cys) for several washing cycles, whereas for a disposable application a single covalent mechanism of grafting L-Cys proved to be sufficient (SF-g-L-Cys).

\section{Acknowledgments}

The authors would like to acknowledge the Fundação para a Ciência e Tecnologia (FCT) for the funding granted concerning the project - PTDC/EBB-BIO/113671/2009 (FCOMP-01-0124-FEDER014752) Skin2Tex and Fundo Europeu de Desenvolvimento Regional (FEDER) through COMPETE - Programa Operacional Factores de Competitividade (POFC) for the co-funding.

The authors would also like to acknowledge the Fundação para a Ciência e Tecnologia (FCT) for the funding for the PhD grant SFRH/BD/91444/2012.

\section{References}

[1] Y.M. Magoshi, M.A. Becker, S. Nakamura, in: J.C. Salamone (Ed.), Biospinning (Silk Fiber Formation, Multiple Spinning Mechanisms), CRC Press, New York, 1996, p. 667

[2] K. Tanaka, N. Kajiyama, K. Ishikura, S. Waga, A. Kikuchi, K. Ohtomo, et al., Determination of the site of disulfide linkage between heavy and light chains of silk fibroin produced by Bombix mori, Biochim. Biophys. Acta 1432 (1) (1999) 92-103.

[3] C.Z. Zhou, F. Confalonieri, N. Medina, Y. Zivanovic, C. Esnault, T. Yang, et al., Fine organization of Bombyx mori fibroin heavy chain gene, Nucleic Acids Res. 28 (12) (2000) 2413-2419.

[4] T. Asakura, K. Suita, T. Kameda, S. Afonin, A.S. Ulrich, Structural role of tyrosine in Bombyx mori silk fibroin, studied by solid-state NMR and molecular mechanics on a model peptide prepared as silk I and II, Magn. Reson. Chem. 42 (2004) 258-266

[5] S.J. He, R. Valluzzi, S.P. Gido, Silk I structure in Bombyx mori silk foams, Int. J. Biol. Macromol. 24 (2-3) (1999) 187-195.

[6] T. Asakura, J.M. Yao, T. Yamane, K. Umemura, A.S. Ulrich, Heterogeneous structure of silk fibers from Bombyx mori resolved by C-13 solid-state NMR spectroscopy, J. Am. Chem. Soc. 124 (30) (2002) 8794-8795.

[7] M. Lewin, Handbook of Fiber Chemistry, 3rd ed., CRC/Taylor \& Francis, Boca Raton, 2007.

[8] F. Arcangeli, M. Feliciangeli, M. Pierleoni, Indumenti di seta nella dermatite atopica. V Convegno. Nazionale Dermatologia per il Pediatra, Bellaria, 2001, pp. 100-101.

[9] N. Bendsoe, A. Bjornberg, H. Asnes, Itching from wool fibres in atopic dermatitis, Contact Dermat. 17 (1987) 21-22

[10] R. Sugihara, M. Yoshimura, M. Mori, N. Kanayama, M. Hikida, H. Ohmori, Prevention of collagen-induced arthritis in DBA/1 mice by oral administration of AZ-9, a bacterial polysaccharide from Klebsiella oxytoca, Immunopharmacology 49 (2000) 325-333.

[11] D.Y.M. Leung, T. Bieber, Atopic dermatitis, Lancet 361 (9352) (2003) 151-160

[12] S. Romagnani, The role of lymphocytes in allergic disease, J. Allergy Clin. Immunol. 105 (2000) 399-408.

[13] C. Hauser, et al., The immune response to S. aureus in atopic dermatitis, Acta Derm. Venereol. Suppl. 114 (1985) 101-104.

[14] J.C. Bystryn, C. Hyman, Skin blood flow in atopic dermatitis, J. Investig. Dermatol. 52 (1969) 189-192.

[15] U.C. Hipler, P. Elsner (Eds.), Biofunctional Textiles and the Skin, Curr. Probl. Dermatol., vol. 33, Karger, Basel, 2006.

[16] C. Hauser, et al., Staphylococcus aureus skin colonization in atopic dermatitis patients, Dermatologica 170 (1985) 35-39.

[17] S.1. Loncarevic, H.J. Jørgensen, A. Løvseth, T. Mathisen, L.M. Rørvik, Diversity of Staphylococcus aureus enterotoxin types within single samples of raw milk and raw milk products, J. Appl. Microbiol. 98 (2) (2005) 344-350.

[18] M.1. Mempel, C. Schnopp, M. Hojka, H. Fesq, S. Weidinger, M. Schaller, H.C. Korting, J. Ring, D. Abeck, Invasion of human keratinocytes by Staphylococcus aureus and intracellular bacterial persistence represent 
haemolysin-independent virulence mechanisms that are followed by features of necrotic and apoptotic keratinocyte cell death, Br. J. Dermatol. 146 (6) (2002) 943-951.

[19] F. Nogueira, C. Mouro, E. Piskin, I. Gouveia, Covalent modification of cellulosic-based textiles: a new strategy to obtain antimicrobial properties, Biotechnol. Bioprocess Eng. 19 (3) (2014) 526-533.

[20] E. Caldeira, E. Piskin, L. Granadeiro, F. Silva, I.C. Gouveia, Biofunctionalization of cellulosic fibers with L-cysteine: assessment of antibacterial properties and mechanism of action against S. aureus and K. pneumoniae, J. Biotechnol. 168 (2013) 426-435.

[21] M.K. Haslekås, P.E. Grini, V. Nygaard, S.H. Nordgard, T.J. Meza, R.B. Aalen, Seed 1 -cysteine peroxiredoxin antioxidants are not involved in dormancy, but contribute to inhibition of germination during stress, Plant Physiol. 133 (3) (2003) 1148-1157.

[22] W. Brand-Williams, C. Berset, Use of a free radical method to evaluate antioxidant activity, Food Sci. Technol. 28 (1) (1995) 25-30.

[23] I.C. Gouveia, D. Sa, M. Henriques, Functionalization of wool with l-cysteine: process characterization and assessment of antimicrobial activity and cytotoxicity, J. Appl. Polym. Sci. 124 (2012) 1352-1358.

[24] G. Socrates, Infrared and Raman Characteristic Group Frequencies: Tables and Charts, John Wiley \& Sons, UK, 2004.

[25] J. Dyer, A. Grosvenor, Protein fibre surface modification, in: E.A. Kumbasar (Ed.), Natural Dyes, 2011.

[26] H.A. Rutherford, W.I. Patterson, M. Harris, Reaction of silk fibroin with diazomethane, J. Res. Natl. Bur. Stand 25 (1940) 451-458.

[27] C.F. Lane, Sodium cyanoborohydride - a highly selective reducing agent for organic functional groups, Synthesis 3 (1975) 135-146.
[28] S.L.W.R. Morris, J.N. Hansen, Identification and characterization of some bacterial membrane sulfhydryl groups which are targets of bacteriostatic and antibiotic action, J. Biol. Chem. 259 (1984) 13590-13594.

[29] Y.H. Kim, C.W. Nam, J.W. Choi, J.H. Jang, Durable antimicrobial treatment of cotton fabrics using N-(2-hydroxy)propyl-3-trimethylammonium chitosan chloride and polycarboxylic acids, J. Appl. Polym. Sci. 88 (6) (2003) 1567-1572.

[30] A. Cottell, S.P. Denyer, G.W. Hanlon, D. Ochs, J.Y. Maillard, Triclosan-tolerant bacteria: changes in susceptibility to antibiotics, J. Hosp. Infect. 72 (1) (2009) 71-76.

[31] S.M. San Miguel, L.A. Opperman, E.P. Allen, J. Zielinski, K.K. Svoboda, Antioxidant compounds increased wound healing migration via Rac-GTP activation in nicotine-treated human gingival and PDL fibroblasts, J. Periodontol. 81 (11)(2010) 1675-1690.

[32] C.L.J. Sanchez-Moreno, F. Saura-Calixto, A procedure to measure the antiradical efficiency of polyphenols, J. Sci. Food Agric. 76 (1998) 270-276.

[33] J. Ji, W. Zhang, Bacterial behaviors on polymer surfaces with organic and inorganic antimicrobial compounds, J. Biomed. Mater. Res. A. 88 (2) (2009) 448-453.

[34] H. Yang, Y. Deng, Preparation and physical properties of superhydrophobic papers, J. Coll. Interface Sci. 325 (2) (2008) 588-593.

[35] V.S.P.C. Hornsey, D.S. Pepper, Reductive amination for solid-phase coupling of protein. A practical alternative to cyanogen bromide, J. Immunol. Methods 93 (1986) 83-88. 